\title{
Hidden Star Formation in High-Velocity Gas Clouds in Clump 2 near the Edge of the CMZ
}

\author{
Volker Tolls, Howard A. Smith and HIGGS Team \\ Smithsonian Astrophysical Observatory \\ 60 Garden Street \\ Cambridge, MA 02478, U.S.A. \\ email: vtolls@cfa.harvard.edu
}

\begin{abstract}
We present a snapshot of our ongoing investigation of molecular clouds in Clump 2 located in the Galactic Bar region at a projected distance of $\sim 400 \mathrm{pc}$ from the Galactic Center. We show that the analysis of the Clump 2 molecular clouds is complicated because of many fore- and background clouds in the line of sight. Of all clouds, IGGC 22 is the most interesting one, showing very high dust column densities, significant high-J CO emission, and, potentially harbors star formations as eluded to by the detection of [OIII] emission.
\end{abstract}

Keywords. Galaxy: center, Galaxy: kinematics and dynamics, Galaxy: structure, ISM: clouds, ISM: kinematics and dynamics, stars: formation

\section{Overview}

The inner few hundred parsecs of the Galaxy are dominated by the Central Molecular Zone (CMZ) containing the densest concentration of gas and dust in the Galaxy (c.f. Stark 2004, Bally 2010, Molinari 2011, Etxaluze 2011, Kudo 2010) at the very center of which lies a massive black hole. Farther out from the center, to about 400 parsecs, is the region called Inner Galaxy (IG) whose dynamics are dominated by the gravitational potential of the Galactic Bar. Material that slowly falls from the outer parts of the Galaxy towards the CMZ encounters extreme physical conditions. Dust and molecular material form dense massive clouds, the so-called Inner Galactic Gas Clumps (IGGC, Martin 2010), but star-formation in them has been suppressed compared to other starforming regions in the Galaxy. The Herschel Inner Galaxy Gas Survey (HIGGS) observed [CII], [NII], [OI], [OIII], and high-J CO emission lines in selected regions of IG Clump 2 with Herschel/HIFI; These data are complemented by Herschel PACS and SPIRE photometry, Spitzer Space Telescope IRAC and MIPS, APEX/ATLASGAL (Schuller 2009), and BOLOCAM data (Ginsburg 2013). We also have a large, new set of molecular line observations of the Clumps obtained with MOPRA and APEX, supplementing existing JCMT and AST/RO spectroscopy data sets.

\section{Observations}

Clump 2. Figure 1 shows a map of Clump 2 in CO J=3-2 (JCMT archival data). Overlaid are the HIGGS fields observed with Herschel HIFI and PACS. The sources IGGC 15, 18, 19, and 21 (their positions are listed in Table 1) form a ridge of clouds with $0 \mathrm{~km} \mathrm{~s}^{-1}<v_{L S R}<80 \mathrm{~km} \mathrm{~s}^{-1}$ from the north east to the south west, while there is a second, almost perpendicular ridge on the northern edge of Clump 2 that ends with IGGC 16. Comparing the velocities with the model by Rodriguez-Fernandez 2008, these 


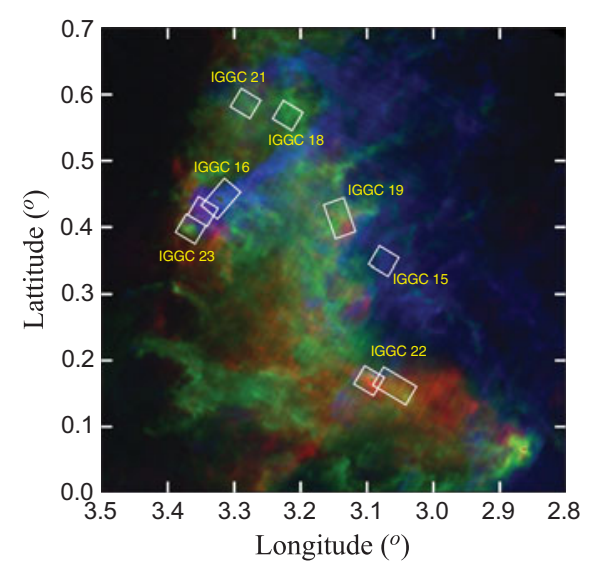

Figure 1: Clump 2: JCMT CO J=3-2.
Table 1. Clump 2 Sources and their positions as identified by AST/RO (Martin 2010) CO J=7-6 observations.

\begin{tabular}{cccc} 
ID & $l$ & $b$ & $\begin{array}{c}\mathbf{v}_{L S R} \\
k m s^{-1}\end{array}$ \\
\hline IGGC 15 & 3.073 & 0.348 & 25.0 \\
IGGC 16 & 3.304 & 0.425 & 41.3 \\
IGGC 17 & 3.277 & 0.613 & 44.7 \\
IGGC 18 & 3.218 & 0.568 & 75.2 \\
IGGC 19 & 3.160 & 0.400 & 83.2 \\
IGGC 20 & 3.205 & 0.614 & 99.9 \\
IGGC 21 & 3.282 & 0.587 & 102.6 \\
IGGC 22 & 3.056 & 0.159 & 146.1 \\
IGGC 23 & 3.353 & 0.394 & 147.5 \\
IGGC 24 & 3.150 & 0.771 & 107.0 \\
\hline
\end{tabular}

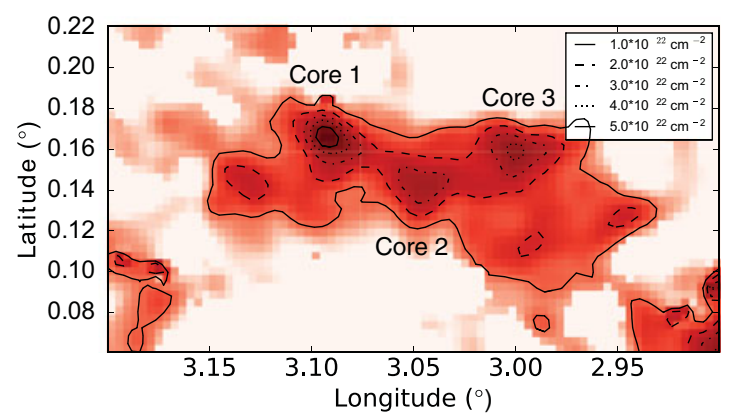

Figure 2: Column density map of IGGC 22. The map shows three distinctive peaks in the column density, Cores 1, 2, and 3 .

clouds could be foreground clouds, possibly part of the near 4-kpc arm. The central area of Clump 2 with $3.1<l<3.4$ and $0.1<b<0.5$, including IGGC 23, harbors clouds primarily with $80 \mathrm{~km} \mathrm{~s}^{-1}<v_{L S R}<160 \mathrm{~km} \mathrm{~s}^{-1}$, which could be located in the far 4$\mathrm{kpc}$ arm, and the southern area clouds including IGGC 22 between $b=0.1-0.2$ have $160 \mathrm{~km} \mathrm{~s}^{-1}<v_{L S R}<240 \mathrm{~km} \mathrm{~s}^{-1}$, which are clouds in the Galactic Bar region.

IGGC 22. The largest dust column densities have been measured around IGGC 22 . The map shown in 2 reveals denser 3 cores in IGGC 22, tagged with Core 1, 2, and 3 . Upper limits for the masses of these cores are $\sim 7500, \sim 13500$, and $\sim 11400 \mathrm{Msol}$ for Core 1,2 , and 3 , respectively. Core 1 is the most interesting densification because it is not only the area with the highest dust column density, but has also significant CO $\mathrm{J}=7-6$ emission centered at the dust peak and to the south of the peak we detected [O III] emission. The analysis of these detections will be presented in an upcoming paper.

\section{References}

Bally, J., et al. 2010 ApJ, 721, 137

Etxaluze, M, et al. 2011 ApJ, 142, 134

Kudo, N, Torii, K, \& Machida, M 2010 PASJ, 63, 171

Martin, C.L. et al. 2010 priv. Comm.

Molinari, S. et al. 2011 ApJ, 735, L33

Rodriguez-Fernandez, N. J. \& Combes, F 2008 A $\& A$, 489, 115

Stark, A. A., et al. 2004, ApJ, 614, L41 